D.T. Wickramasinghe, L. Ferrario, and G.V. Bicknell, eds.

\title{
HST GHRS Observations of the Herbig Ae Star HD104237: First UV Observations of a Hot Disk Wind from a Pre-Main Sequence Star
}

\author{
Alexander Brown ${ }^{1}$, H.R.E. Tjin A Djie ${ }^{2}$, P.F.C. Blondel ${ }^{2}$, G.M. \\ Harper $^{1}$, P.D. Bennett ${ }^{1}$, \& S.L. Skinner ${ }^{3}$ \\ ${ }^{1}$ Center for Astrophysics and Space Astronomy, Campus Box 389, \\ University of Colorado, Boulder, Colorado 80309-0389, USA \\ ${ }^{2}$ Astronomical Institute "Anton Pannekoek", University of Amsterdam, \\ Kruislaan 403, 1098 SJ Amsterdam, The Netherlands \\ ${ }^{3}$ JILA, University of Colorado, Boulder, Colorado 80309-0440, USA
}

\begin{abstract}
We have obtained ultraviolet spectra of the Herbig Ae star HD104237 using the Goddard High Resolution Spectrograph (GHRS) on HST. The high temperature emission and absorption lines show remarkable outflow absorption features, which have very similar profiles that are essentially independent of formation temperature. The profiles are not those expected from a spherically-symmetric stellar wind but have optically-thick absorption to $-280 \mathrm{~km} \mathrm{~s}^{-1}$ and a high velocity plateau extending to $-375 \mathrm{~km} \mathrm{~s}^{-1}$. The profile shape is a manifestation of the nonspherical geometry of the flow. The observed UV emission is too strong to be associated with the hot X-ray emitting plasma seen by ASCA and probably is generated by the interaction of the innermost part of an accretion disk with the corotating outermost magnetospheric field. The outflow is almost certainly the inner part of a biconical disk wind.
\end{abstract}

\section{Introduction}

Herbig Ae/Be stars are early-type emission line stars that lie in T Tauri associations, star formation regions and young open clusters. They are intermediate mass $\left(2-8 \mathrm{M}_{\odot}\right)$ stars undergoing radiative equilibrium contraction towards the main sequence. Like their lower mass counterparts the T Tauri stars, these stars are surrounded by extended gas and dust which are revealed by strong far- and near-infrared excesses and broad asymmetric emission and absorption lines in the visual and ultraviolet. Strong chromospheric and transition region (TR) emission lines are seen below $1600 \AA$ in the IUE and HST/GHRS spectra of later-type (A3 and later) Herbig emission line stars (see Fig. 1).

The origin of the UV emission lines from Herbig Ae stars is a topic of considerable argument. Palla and Stahler (1990) discussed ways that the heating of such hot regions might be powered by an outer convective zone caused by deuterium burning and produce a relatively normal chromosphere and TR. Others have suggested that the emission is due to accretion onto the stellar surface 

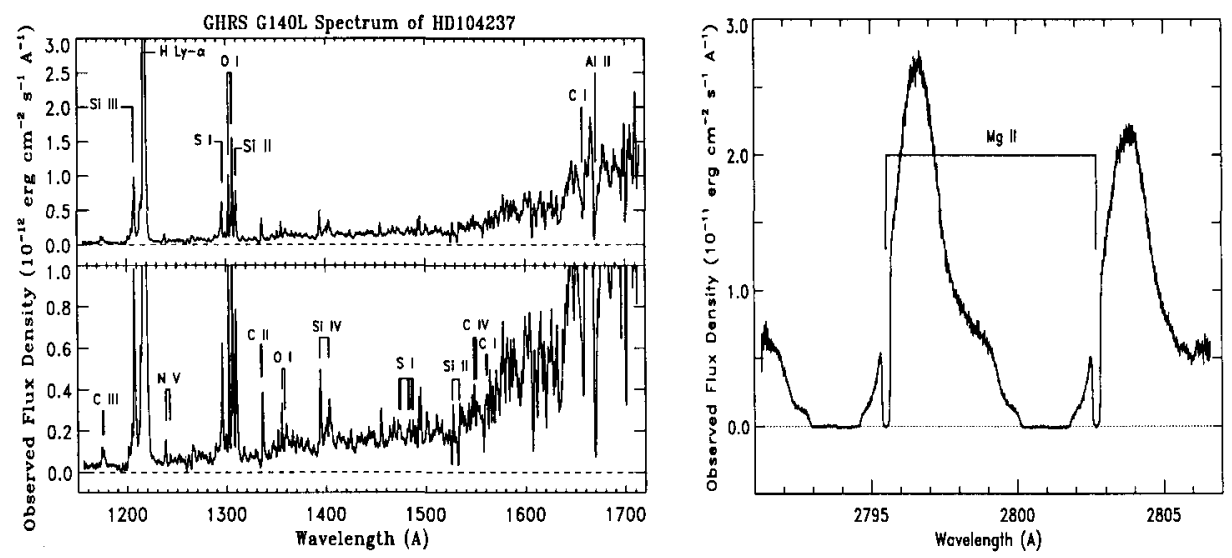

Figure 1. GHRS G140L spectra (left) and Ech-B Mg II spectra (right) of HD104237

(e.g. Blondel et al., 1993). Shu et al. (1994) presented a model for magnetocentrifugally driven outflows from young stars that we feel accounts for most of the properties we observe in the UV spectra of Herbig Ae stars, while Grinin \& Rostopchina (1996) outlined a scenario that explains the observed dynamic juxtaposition of infall and outflow as the result of observing a disk/outflow configuration at different viewing angles.

\section{Observations}

We obtained GHRS low, medium and echelle resolution observations of HD104237 (A7 IVe) on 1994 November 7, which severely constrain the physical conditions in the regions close to this star. The GHRS spectra provide information on both the hot boundary layer line and continuum emission, and the mass outflow.

Figure 1 shows that numerous emission lines are present in the G140L spectrum; these lines range in excitation from $\mathrm{O}$ I to $\mathrm{N} \mathrm{V}$ with all the emission lines expected from temperatures of few $10^{3}$ to $\sim 10^{5} \mathrm{~K}$ being detected. At longer wavelengths, above $1500 \AA$, the emission lines are subsumed by the rapidly rising photospheric continuum, and most of the high temperature lines in this region are seen only in absorption. Below $1500 \AA$ continuum emission is evident but this is not photospheric; continuum fitting suggests temperatures of at least $10-2010^{3} \mathrm{~K}$. While the hot continuum may originate where accreting matter strikes the stellar surface, there is strong evidence that the emission lines form well above the surface where the stellar and disk magnetic fields interact. If the emission lines form in such a "boundary layer" they suggest maximum temperatures approaching $10^{5} \mathrm{~K}$. Skinner and Yamauchi (1996) observed strong X-ray emission from HD104237 with temperatures of few $10^{6}$ - few $10^{7} \mathrm{~K}$. Thus, a hot corona most likely surrounds the Herbig star and may be a spatially-organised magnetosphere. Magnetic reconnection between the magnetosphere and disk fields could be the heating source for the boundary layer and hot X-ray plasma. The relative luminosities of the TR emission lines (e.g. Si IV) and the X-rays 


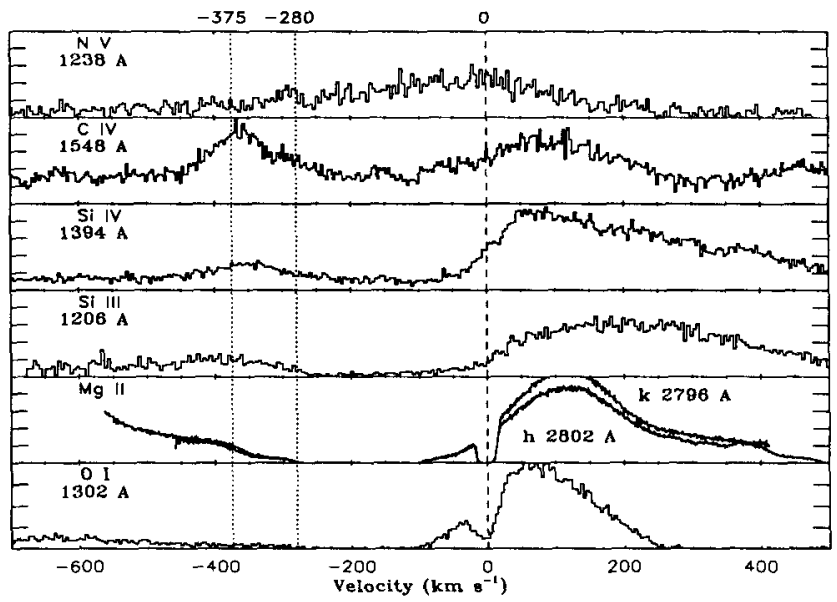

Figure 2. The wind absorption profiles of HD104237 seen against a variety of emission lines. Note that absorption is seen over the same velocity range for all the lines. The C IV absorptions are seen against the photospheric continuum; the dip at $+200-400 \mathrm{~km} \mathrm{~s}^{-1}$ is due to the second C IV line at $1550 \AA$. The long-wavelength sides of the O I $1302 \AA$ and the $\mathrm{Mg}$ II k lines are eaten into by other multiplet members. Only outflow signatures are seen; there is no sign of inverse $\mathrm{P}$ Cygni profiles indicative of infalling material.

are not those expected from the TR/coronal scaling laws derived from normal late-type stars; the UV lines are eight times too bright to come from a normal $\mathrm{TR}+$ corona.

The Mg II emission profiles (Fig. 1) demonstrate another significant feature: strong blue-shifted absorption lines are superimposed on almost all the emission lines. The Mg II absorption profile shows a narrow interstellar line at zero velocity and a wind line that is black from $\sim-80 \mathrm{~km} \mathrm{~s}^{-1}$ to $-280 \mathrm{~km} \mathrm{~s}^{-1}$. The profile then rises to a plateau, covering about $40 \mathrm{~km} \mathrm{~s}^{-1}$, which eventually rises again to the local continuum level at about $-375 \mathrm{~km} \mathrm{~s}^{-1}$. Figure 2 shows the emission profiles for a variety of emission lines and careful examination of the absorption features shows that the wind absorptions have the same velocity structure independent of line formation temperature. While wind scattering must be contributing some of the red emission features, profile fitting shows that intrinsic emission components are present. The hot lines, e.g. Si III-IV, C IV, have absorptions that are not black indicating that either the continuum source is not fully obscured or the lines are not very optically thick.

The emission lines are extremely broad with uncontaminated lines showing FWZI of $\geq 800 \mathrm{~km} \mathrm{~s}^{-1}$. The photospheric $\mathrm{v} \sin i$ is $180 \mathrm{~km} \mathrm{~s}^{-1}$ so the line widths are not due to photospheric rotation. Turbulence is not a likely source of line broadening at the $400 \mathrm{~km} \mathrm{~s}^{-1}$ level. However, if the boundary layer stands well off the stellar surface and the magnetosphere rotates rigidly, the emission line broadening might be dominated by rotation and the distance between the boundary layer and the stellar surface would be $\sim 1 \mathrm{R}_{*}$. 


\section{Discussion}

Given the above observed properties we can construct a schematic model of the region surrounding HD104237, which should be relevant also to other Herbig Ae stars. The components of this schematic model are a) at the center the star providing the optical and near UV photospheric continuum, b) a magnetosphere filled with coronal gas immediately surrounding the star and extending $\sim 1 \mathrm{R}_{*}$ above the photosphere, c) an accreting circumstellar disk, that is cool, molecular, and dusty far from the star but ionized in the inner regions, d) a boundary layer at the interface between the disk and magnetosphere that is most likely heated by magnetic reconnection and is an important heating source for its immediate surroundings, and e) a biconical outflow moving generally perpendicular to the disk plane. The outflow should probably be imagined as a set of nested bicones whose temperature decreases with increasing distance from the star in the disk plane. Such nested cones would mimic the fact the flows at different temperatures can have the same basic velocity distributions; this behavior is not possible with a single temperature at each point on the velocity law. HD104237 is viewed at about $50^{\circ}$ to the stellar rotational axis, based on disk modelling and the stellar $\mathrm{v} \sin i$. In such a geometry the front and back sides of the unobscured cone will show different velocities; one at $V_{o} \cos (i-\theta)$ and the other at $V_{o}$ $\cos (i+\theta)$, where $V_{o}$ is the flow velocity within the cone and $\theta$ is the opening (or closing angle) of the bicone. We observe wind absorption edges near -375 and $-280 \mathrm{~km} \mathrm{~s}^{-1}$; these values have not yet been adjusted for turbulent broadening but this correction would mostly effect $V_{o}$. Taken at face value these velocities imply $V_{o}=500 \mathrm{~km} \mathrm{~s}^{-1}$ and $|\theta|=7^{\circ}$. Thus, the flow is already highly collimated in the region where it is being formed. This opening angle could in principle describe either a converging or diverging flow. Because the higher velocity wind absorption obscures less of the underlying continuum, we suspect that the flow is actually converging. Clearly UV spectra offer the opportunity to study the innermost parts of bipolar outflows, and perhaps allow detailed study of the collimation process. Such observational results provide vital data for theoretical modelling of accretion onto young stars and offer insights into accretion phenomena that may be useful for understanding the wide range of objects discussed at this conference.

Acknowledgments. Support for this work was provided by NASA through grants GO-5495.01-93A and GO-0539.01-93A from the Space Telescope Science Institute, which is operated by AURA, Inc., under NASA contract NAS 5-26555.

\section{References}

Blondel, P.F.C., Talavera, A., \& Tjin A Djie, H.R.E. 1993, A\&A, 268, 624

Grinin, V.P. \& Rostopchina, A.N. 1996, Ast. Rep., 40, 194

Palla, F. and Stahler, S.W. 1990, ApJ, 360, L47

Shu, F. et al. 1994, ApJ, 429, 781

Skinner, S.L. \& Yamauchi, S. 1996, ApJ, 471, in press 


\section{Discussion}

G. Israelian: How has the rotational velocity of the star been derived? Could it be that lines used to derive $v \sin i$ were filled in by some emission?

A. Brown: The optical spectrum shows only very shallow absorption lines and the $\mathrm{H} \alpha$ line shows a strong emission core. The measured rotational velocity is based primarily on the MgII $4481 \AA$ absorption profile.

$U$. Torkelsson: There is nothing surprising in there being reconnection events in the interaction region between the stellar magnetosphere and the disc. A stellar dynamo in general generates a dipolar magnetic field, whereas the disk dynamo generates a quadrupolar magnetic field. The stellar and disc magnetic fields are thus topologically different and reconnection must occur.

A. Brown: I agree that magnetic reconnection in inevitable. I only emphasise the point here because this important physical process is ignored in most current PMS disk models.

H. Zinnecker: As far as the X-Rays from the HD 104237 system are concerned, I must caution that the $\mathrm{X}$-Rays might come from a close $\mathrm{T}$ Tauri-like companion that we recently discovered by infrared adaptive optics observations at the ESO 3.6 in telescope.

A. Brown: Skinner and Yamauchi considered the possible influence of this fainter star on their ASCA spectra. Based on the observed luminosity and spectral distribution, they concluded that at best this star could only be a partial contributor to the X-Ray flux. Additionally, the theoretical models that they considered did not include the possibility that magnetic reconnection between stellar and disk fields might be an important source of hot plasma. 